\title{
ACCELERATION-EXTENDED GALILEAN SYMMETRIES WITH CENTRAL CHARGES AND THEIR DYNAMICAL REALIZATIONS*
}

\author{
J. Lukierski ${ }^{1)}$, P.C. Stichel ${ }^{2)}$ and W.J. Zakrzewski ${ }^{3)}$ \\ 1) Institute for Theoretical Physics, University of Wrocław, \\ pl. Maxa Borna 9, 50-205 Wrocław, Poland \\ e-mail: lukier@ift.uni.wroc.pl \\ 2) An der Krebskuhle 21, D-33619 Bielefeld, Germany \\ e-mail:peter@physik.uni-bielefeld.de \\ ${ }^{3)}$ Department of Mathematical Sciences, University of Durham, \\ Durham DH1 3LE, UK \\ e-mail: W.J.Zakrzewski@durham.ac.uk
}

\begin{abstract}
We add to Galilean symmetries the transformations describing constant accelerations. The corresponding extended Galilean algebra allows, in any dimension $D=d+1$, the introduction of one central charge $c$ while in $D=2+1$ we can have three such charges: $c, \theta$ and $\theta^{\prime}$. We present nonrelativistic classical mechanics models, with higher order time derivatives and show that they give dynamical realizations of our algebras. The presence of central charge $c$ requires the acceleration square Lagrangian term. We show that the general Lagrangian with three central charges can be reinterpreted as describing an exotic planar particle coupled to a dynamical electric and a constant magnetic field.
\end{abstract}

${ }^{*}$ Supported by the KBN grant 1P03B01828 


\section{Introduction}

In our recent paper [1] we studied conformal extensions of Galilean symmetries in $D=2+1$ dimensions. Our extension was performed by the addition of four generators:

i) two generators - dilation $D$ and expansion $K$ - extending the Galilean algebra to the Schrödinger algebra [2-4]

ii) two new generators $F_{i},(i=1,2)$ describing constant accelerations.

It is easy to see that such an extension can be made for any nonrelativistic dimension $d$; in fact, it can be obtained as a nonrelativistic $c \rightarrow \infty$ limit of the relativistic $D$-dimensional $(D=d+1)$ conformal algebra described by the Lie algebra $O(d, 2)^{1}$. In such a derivation the generators $F_{i}$ $(i=1 \ldots d)$ are provided by the nonrelativistic limits of space components of special conformal generators and describe, in a nonrelativistic theory, constant accelerations. Thus, such acceleration-extended Galilean symmetries, in $d$ spacial dimensions, are described by $\frac{1}{2} d(d-1)+3 d+1$ generators, namely $(i=1 \ldots d)$

- $J_{i j}=-J_{j i}\left(\right.$ space rotations $\left.\alpha_{i j}\right)$

- $P_{i}$ (space translations $a_{i}$ )

- $K_{i}$ (constant velocity motions, or Galilean boosts $v_{i}$ )

- $F_{i}$ (constant acceleration motions, or Galilean accelerations $b_{i}$ )

- $H$ (time translations $\left.t^{\prime}=t+a\right)$.

These symmetries taken in their infinitesimal form can be realised in nonrelativistic space-time as

$$
\begin{gathered}
\delta x_{i}=-\delta a_{i}-\delta v_{i} t-\delta b_{i} t^{2}+\delta \alpha_{i j} x_{j}, \\
\delta t=\delta a .
\end{gathered}
$$

The acceleration-extended Galilean algebra has the following nonvanishing commutators:

$$
\begin{gathered}
{\left[J_{i j}, J_{k l}\right]=\delta_{i k} J_{j l}-\delta_{j l} J_{i k}+\quad(i \leftrightarrow j, k \leftrightarrow l),} \\
{\left[J_{i j}, A_{k}\right]=\delta_{i k} A_{j}-\delta_{j k} A_{i} \quad\left(A_{i}=P_{i}, K_{i}, F_{i}\right),} \\
{\left[H, K_{i}\right]=P_{i}}
\end{gathered}
$$

\footnotetext{
${ }^{1}$ In [1] such a limit was mentioned for $D=2+1$
} 


$$
\left[H, F_{i}\right]=2 K_{i} .
$$

The aim of this paper is to study central extensions of the algebra (2-3) and its dynamical representations.

First we recall that the standard Galilean algebra (relations (2) for $J_{i j}, P_{i}, K_{i}$ and $H$ ) has two central extensions:

i) For arbitrary $D$ one can introduce one central extension [5]

$$
\left[P_{i}, K_{j}\right]=m \delta_{i j},
$$

where $m$ describes the nonrelativistic mass parameter.

ii) In $D=2+1$ we can introduce a second charge $\theta$, called exotic [6], [7]

$$
\left[K_{i}, K_{j}\right]=\theta \epsilon_{i j},
$$

which can be related to the noncommutativity of the space components of the nonrelativistic $D=2+1$ space-time [8].

A dynamical realisation of the $D=2+1$ exotic algebra can be deduced from the following nonrelativistic model [8]

$$
L=\frac{1}{2} m \dot{x}_{i}^{2}-\frac{\theta}{2} \epsilon_{i j} \dot{x_{i}} \ddot{x_{j}} .
$$

One can show by considering the Jacobi identity for the three generators $H, P_{i}, F_{j}$ and using (3), (4) $\left(0 \equiv\left[H,\left[P_{i}, F_{j}\right]\right]+\left[P_{i}\left[F_{j}, H\right]\right]+\left[F_{j},\left[H, P_{i}\right]\right]=\right.$ $\left.-2\left[P_{i}, K_{j}\right]=-2 m \delta_{i j}\right)$ that in acceleration - extended Galilei algebra we must put $m=0$. In such a case one arrives at the following central extensions

- For arbitrary $D$ one can introduce a single central extension

$$
\left[K_{i}, F_{j}\right]=2 c \delta_{i j} .
$$

- In $D=(2+1)$, in addition to relations (5) and (7) one can have a third central charge

$$
\left[F_{i}, F_{j}\right]=\theta^{\prime} \epsilon_{i j} .
$$

The algebraic consistency requires also that

$$
\left[P_{i}, F_{j}\right]=-2 \theta \epsilon_{i j} .
$$

The paper is organised as follows. In the next section we consider a dynamical model in any dimension $D$ with the Lagrangian being given by the square of the accelerations of the particle and the Noether charges satisfying (7). In Section 3 we discuss those properties of the acceleration-extended $D=2+1$ Galilean algebra which are model independent, $i e$ have a purely 
algebraic origin. We consider the enveloping algebra $U\left(\hat{g}_{\theta}\right)$ with one central charge $\theta$ (see (5) and (9)) and we show that the symmetry algebras with three central charges can be embedded in $U\left(\hat{g}_{\theta}\right)$. We also present the Casimirs in the presence of central charges and discuss the possible enlargements of the symmetry algebra. A particular case here is the extension of the Galilean conformal algebra by the addition of two further generators $D$ and $K$. We find that when all three central parameters $\left(\theta, \theta^{\prime}\right.$ and $\left.c\right)$ are nonzero, the commutators in the Galilean conformal algebra involving the generators $D$ and $K$ are deformed. In Section 4 we introduce $D=2+1$ Lagrangian models which realize this three-fold centrally extended symmetry algebra. In particular, in Section 4.3, we show that the general planar model with three central charges can be reinterpreted as describing the motion of a planar noncommutative particle interacting with dynamic electric and constant magnetic fields. Section 5 contains some concluding remarks.

\section{An acceleration square Lagrangian and a new central charge $c$ (arbitrary $D=d+1)$}

The nonrelativistic kinetic term which is quasi-invariant under the acceleration extended Galilei symmetry $(1)$ has the form $\left(\ddot{x}^{2}=\ddot{x}_{i} \ddot{x}_{i} ; i=1, . . d\right)$

$$
L=\frac{c \ddot{x}^{2}}{2} .
$$

Indeed, performing the transformation (1) one obtains

$$
\delta L=-2 c \delta b_{i} \ddot{x}_{i}=\frac{d}{d t}\left(-2 c \delta b_{i} \dot{x}_{i}\right) .
$$

Introducing $y_{i}=\dot{x}_{i}$ as an independent coordinate the Lagrangian (10) can be put into the following equivalent first order form

$$
L=p_{i}\left(\dot{x}_{i}-y_{i}\right)+q_{i} \dot{y}_{i}-\frac{1}{2 c} q_{i}^{2} .
$$

Using the Faddeev-Jackiw procedure [9]-[10] we obtain the following nonvanishing Poisson brackets

$$
\left\{x_{i}, p_{j}\right\}=\delta_{i j}, \quad\left\{y_{i}, q_{j}\right\}=\delta_{i j} .
$$

The acceleration-extended Galilean transformations of the additional variables $\left(p_{i}, y_{i}, q_{i}\right)$, which are consistent with the field equations

$$
y_{i}=\dot{x}_{i}, \quad \dot{p}_{i}=0,
$$




$$
\dot{y}_{i}=\frac{1}{c} q_{i}, \quad \dot{q}_{i}+p_{i}=0
$$

take the form

$$
\begin{gathered}
\delta p_{i}=\delta \alpha_{i j} p_{j} \\
\delta y_{i}=-\delta v_{i}-2 t \delta b_{i}-\frac{\delta a}{c} q_{i}+\delta \alpha_{i j} y_{j} \\
\delta q_{i}=-2 c \delta b_{i}+\delta a p_{i}+\delta \alpha_{i j} q_{j}
\end{gathered}
$$

and also

$$
\delta x_{i}=-\delta a_{i}-t \delta v_{i}-t^{2} \delta b_{i}+\delta \alpha_{i j} x_{j}-y_{i} \delta a .
$$

We note that the variation (15, 16) implies that the Lagrangian is quasiinvariant

$$
\delta L=\frac{d}{d t}\left(\delta a p_{i} y_{i}-2 c \delta b_{i} y_{i}\right) .
$$

The transformations (15, 16) are generated by the Poisson brackets (13) in the standard way ${ }^{2}$ by the following realization of the generators of the acceleration extended algebra with one central charge $c$ (see (7)):

$$
\begin{gathered}
P_{i}=p_{i}, \quad H=p_{i} y_{i}+\frac{1}{2 c} q_{i}^{2}, \\
J_{i j}=\frac{1}{2}\left(x_{[i} p_{j]}+y_{[i} q_{j]}\right), \\
K_{i}=q_{i}+p_{i} t, \quad F_{i}=-2 c y_{i}+2 t q_{i}+t^{2} p_{i},
\end{gathered}
$$

where the second term in the energy generator $H$ is consistent with Souriau general theorem on the barycentric decomposition of nonrelativistic systems in phase space $[11,12]$. It is easy to check, using (13), that (7) is satisfied.

In quantum theory the quantized Poisson brackets (13) take the form

$$
\left[\hat{x}_{i}, \hat{p}_{j}\right]=i \hbar \delta_{i j}, \quad\left[\hat{y}_{i}, \hat{q}_{j}\right]=i \hbar \delta_{i j}
$$

and can be realised in the standard way on the wave functions $\Psi\left(x_{i}, y_{j}\right)$ via the Schrödinger representation:

$$
\hat{x}_{i}=x_{i}, \quad \hat{p}_{i}=\frac{\hbar}{i} \frac{\partial}{\partial x_{i}}, \quad \hat{y}_{i}=y_{i}, \quad \hat{q}_{i}=\frac{\hbar}{i} \frac{\partial}{\partial y_{i}} .
$$

The Schrödinger equation, in the first quantized theory, corresponding to the Lagrangian (10) and the Hamiltonian (18) takes the form

$$
\left(-\frac{\hbar^{2}}{2 c} \frac{\partial}{\partial y_{i}} \frac{\partial}{\partial y_{i}}+\frac{\hbar}{i} y_{i} \frac{\partial}{\partial x_{i}}\right) \Psi=E \Psi .
$$

\footnotetext{
${ }^{2}$ The variation of the phase space variables $Y_{k}$, generated by $G_{r}$, is given by $\delta Y_{i}=$ $\delta \alpha_{r}\left\{G_{r}, Y_{i}\right\}\left(\alpha_{r}\right.$ - symmetry parameters).
} 


\section{Properties and Enlargements of the $D=$ $2+1$ acceleration-extended Galilean algebra}

The space of dimension $d=2$ is special due to the existence of an antisymmetric covariant constant tensor $\epsilon_{i j}=\left(\begin{array}{cc}0 & 1 \\ -1 & 0\end{array}\right)$. If, for $d=2$, we extend the Galilei algebra by adding to it two generators $F_{i}$, describing constant acceleration motions, due to the existence of the tensor $\epsilon_{i j}$, we can introduce, through relations (5) and (8) two independent central charges $\theta$ and $\theta^{\prime}$. In the general case, for $d=2$, we can introduce three central charges: $\theta, \theta^{\prime}$ and $c$.

\subsection{How do we go from one central charge to three}

Let us observe that the status of all three central charges is not the same. To see this we introduce the universal enveloping algebra $U\left(\hat{g}_{\theta}\right)$, where $\hat{g}_{\theta}$ denotes the acceleration-extended Galilei algebra with only one central charge $\theta$. Then we observe that the following modification of the relations

$$
\begin{aligned}
& {\left[K_{i}, F_{j}\right]=0, \quad \rightarrow \quad\left[K_{i}^{\prime}, F_{j}^{\prime}\right]=2 c \delta_{i j}} \\
& {\left[F_{i}, F_{j}\right]=0, \quad \rightarrow \quad\left[F_{i}^{\prime}, F_{j}^{\prime}\right]=\theta^{\prime} \delta_{i j}}
\end{aligned}
$$

can be achieved by the linear change of basis inside the enveloping algebra $U\left(\hat{g}_{\theta}\right) 3$

$$
\begin{array}{rc}
K_{i}^{\prime}= & K_{i}+\frac{c}{2 \theta} \epsilon_{i j} P_{j} \\
F_{i}^{\prime}= & F_{i}+\frac{c}{\theta} \epsilon_{i j} K_{j}+\frac{1}{4}\left(\frac{c^{2}}{\theta^{2}}-\frac{\theta^{\prime}}{\theta}\right) P_{i} .
\end{array}
$$

It can be checked that under this transformation all Lie algebra relations of $\hat{g}_{\theta}$ besides (24) remain unchanged. Thus we see that the two acceleration extended Galilean algebra with a one parameter central extension (by the parameter $\theta$ ) and the algebra with three central extension parameters $\left(\theta, c, \theta^{\prime}\right)$ have the same enveloping algebras. We can recall here the well known analogy: in $D=2+1$ spacetime the exotic Galilean algebra with two central charges $(m, \theta)$ can be obtained through the transformation [13], [8]

$$
K_{i}^{\prime}=K_{i}-\frac{\theta}{2 m} \epsilon_{i j} P_{j}
$$

where $K_{i}$ and $P_{i}$ belong to the standard Galilean algebra with one central charge $m$ :

$$
\left[K_{i}, K_{j}\right]=\left[P_{i}, P_{j}\right]=0, \quad\left[K_{i}, P_{j}\right]=i \delta_{i j} m .
$$

\footnotetext{
${ }^{3}$ Remaining generators $P_{i}, H, J$, stay unchanged.
} 
Indeed, the generators $\left(K_{i}^{\prime}, P_{i}^{\prime}=P_{i}\right)$ in $D=2+1$ lead to the appearance of an 'exotic' central charge

$$
\left[K_{i}^{\prime}, K_{j}^{\prime}\right]=i \epsilon_{i j} \theta, \quad\left[P_{i}^{\prime}, P_{j}^{\prime}\right]=0, \quad\left[K_{i}^{\prime}, P_{j}^{\prime}\right]=i \delta_{i j} m
$$

which was recently reinterpreted as generating noncommutativity of the $d=$ 2 space coordinates [8], 14].

\subsection{Casimirs}

$\hat{g}_{\theta}$ has two Casimirs:

$$
\begin{aligned}
C_{H} & =H-\frac{1}{\theta} \epsilon_{i j} K_{i} P_{j} \\
\text { and } \quad C_{J} & =J-\frac{1}{2 \theta}\left(F_{i} P_{i}-K_{i}^{2}\right) .
\end{aligned}
$$

The Casimirs for the case of three central charges $\theta, \theta^{\prime}$ and $c$ can be easily obtained from (29) by the transformation (25) with the result

$$
\begin{aligned}
& C_{H}^{\prime}=\quad H-\frac{1}{\theta} \epsilon_{i j} K_{i}^{\prime} P_{j}+\frac{c}{2 \theta^{2}} P_{i}^{2} \\
& \text { and } \quad C_{J}^{\prime}=J-\frac{1}{2 \theta}\left(F_{i}^{\prime} P_{i}-K_{i}^{\prime 2}\right)-\frac{c}{\theta} H-\frac{\theta^{\prime}}{8 \theta^{2}} P_{i}^{2} \text {. }
\end{aligned}
$$

\subsection{Enlargement by an $O(2,1)$ algebra}

We may add to $\hat{g}_{\theta}$ two further generators

$$
J_{ \pm}=\frac{1}{4 \theta}\left(K_{ \pm}^{2}-F_{ \pm} P_{ \pm}\right)
$$

where $K_{ \pm}=K_{1} \pm i K_{2}$ etc. For $C_{J}=0$ they form together with $J$ an $O(2,1)$ algebra

$$
\left[J_{3}, J_{ \pm}\right]=\mp i J_{ \pm}, \quad\left[J_{+}, J_{-}\right]=2 i J_{3},
$$

where $J_{3}=\frac{J}{2}$. The remaining nonvanishing commutators of $J_{ \pm}$describe the $O(2,1)$ covariance of any two-vector $A_{i} \in\left(P_{i}, K_{i}, F_{i}\right)$

$$
\left[J_{+}, A_{-}\right]=-i A_{+}, \quad\left[J_{-}, A_{+}\right]=i A_{-} .
$$

In the Lagrangian model discussed in [1] we had $C_{J}=0$ and so this model provided a realization of this $O(2,1)$ algebra. 


\subsection{Extension to $D=2+1$ conformal Galilean algebra and central charges}

To obtain the Galilean conformal algebra [1] one has to add to the accelerationextended Galilean algebra (2-4) two further generators: dilatation $D$ and expansion $K$, which together with the Hamiltonian, form an $O(2,1)$ subalgebra

$$
[D, H]=-2 H, \quad[D, K]=2 K, \quad[H, K]=D .
$$

The generators $D$ and $K$ are scalars 4

$$
[D, J]=[K, J]=0
$$

and, in addition, they satisfy

$$
\begin{array}{cc}
{\left[D, P_{i}\right]=-P_{i}, \quad\left[D, K_{i}\right]=0, \quad\left[D, F_{i}\right]=F_{i},} \\
{\left[K, P_{i}\right]=-2 K_{i}, \quad\left[K, K_{i}\right]=-F_{i}, \quad\left[K, F_{i}\right]=0 .}
\end{array}
$$

It is easy to argue that the Galilean conformal algebra does not permit the central extensions with parameters $c$ and $\theta^{\prime}$. Indeed, if we observe that from (34-36) we get the following mass dimensions of the generators

$$
\begin{gathered}
{\left[P_{i}\right]=M^{1}, \quad\left[K_{i}\right]=M^{0}, \quad\left[F_{i}\right]=M^{-1}} \\
{[H]=M^{1}, \quad[D]=M^{0}, \quad[K]=M^{-1} \quad[J]=M^{0}}
\end{gathered}
$$

we obtain from (5), (77) and (8) that

$$
[\theta]=M^{0}, \quad[c]=M^{-1}, \quad\left[\theta^{\prime}\right]=M^{-2} .
$$

We see from (38) that the constants $c$ and $\theta^{\prime}$ break the scale and conformal invariance. In fact, if we supplement the transformation (25) by the relations $D^{\prime}=D$ and $K^{\prime}=K$ we get from the generators of the acceleration-extended Galilean algebra $\hat{g}_{\theta}$ with one nonvanishing central charge the following deformation of the conformal Galilean algebra 5

$$
\begin{array}{rcc}
{\left[K^{\prime}, P_{i}^{\prime}\right]} & = & -2 K_{i}^{\prime}+\frac{c}{\theta} \epsilon_{i j} P_{j}^{\prime} \\
{\left[K^{\prime}, F_{i}^{\prime}\right]} & = & -\frac{c}{\theta} \epsilon_{i j} F_{j}^{\prime}-\frac{3}{2} \frac{c^{2}}{\theta^{2}} K_{i}^{\prime}+\frac{1}{\theta^{2}}\left(\frac{c^{2}}{\theta}-\frac{\theta^{\prime}}{2}\right) \epsilon_{i j} P_{j} \\
{\left[K^{\prime}, K_{i}^{\prime}\right]} & = & -F_{i}^{\prime}+\frac{1}{4 \theta}\left(\frac{c^{2}}{\theta}-\theta^{\prime}\right) P_{i}^{\prime} \\
{\left[D^{\prime}, K_{i}^{\prime}\right]} & = & -\frac{c}{2 \theta} \epsilon_{i j} P_{j}^{\prime} \\
{\left[D^{\prime}, F_{i}^{\prime}\right]} & = & F_{i}^{\prime}-\frac{c}{\theta} \epsilon_{i j} K_{j}^{\prime}-\frac{1}{\theta}\left(\frac{c^{2}}{\theta}-\frac{\theta^{\prime}}{2}\right) P_{i}^{\prime} .
\end{array}
$$

\footnotetext{
${ }^{4}$ We recall that in $d=2 J_{i j}=\epsilon_{i j} J$.

${ }^{5} \mathrm{We}$ only list the modified relations.
} 
The parameters $c$ and $\theta^{\prime}$ cease to be central charges and take the role of deformation parameters (see eg [15]). The deformed conformal Galilean algebra with the generators $\left(P_{i}^{\prime}, K_{i}^{\prime}, F_{i}^{\prime}, J^{\prime}, H^{\prime}, D^{\prime}\right)$ and the three parameters $(\theta, c$ and $\left.\theta^{\prime}\right)$ can be treated as a particular choice of basis in the enveloping conformal Galilean algebra $U\left(\hat{g}_{\theta}\right)$ with one central charge.

\section{Dynamical planar models realizing the ac- celeration extended Galilean symmetry with three central charges}

In this section we introduce and discuss $D=2+1$ nonrelativistic bilinear Lagrangian models which possess the acceleration-extended Galilean symmetry with three central charges $\left(\theta, \theta^{\prime}\right.$ and $\left.c\right)$.

\subsection{Higher-order derivative model}

The most general $D=2+1$ Lagrangian which is bilinear in the time derivatives of the coordinates $x_{i}$ and is quasi-invariant under transformations (11) has the form:

$$
L=-\frac{\theta}{2} \epsilon_{i j} \dot{x}_{i} \ddot{x}_{j}+\frac{c}{2} \ddot{x}_{j}^{2}-\frac{\theta^{\prime}}{8} \epsilon_{i j} \ddot{x}_{i} \dot{\dot{x}_{j}} .
$$

The model consisting only of the first term in (40) (i.e. with $\theta \neq 0, \theta^{\prime}=c=$ $0)$ was considered in detail in [1].

The extended Galilean transformations (11) provide the following variation of the Lagrangian (40):

$$
\delta L=\frac{d}{d t}\left(-\frac{\theta}{2} \epsilon_{i j} \dot{x}_{j}\right) \delta v_{i}+\frac{d}{d t}\left[\theta \epsilon_{i j}\left(t \dot{x}_{j}-2 x_{j}\right)-2 c \dot{x}_{i}+\frac{\theta^{\prime}}{4} \epsilon_{i j} \ddot{x}_{j}\right] \delta b_{i} .
$$

The invariance only modulo total derivative (quasi-invariance) implies the existence of central charges defined by the relations (5) and (7, 9 ).

In the first order formalism the Lagrangian (40) then takes the form

$$
L=p_{i}\left(\dot{x}_{i}-y_{i}\right)+q_{i}\left(\dot{y}_{i}-u_{i}\right)-\frac{\theta^{\prime}}{8} \epsilon_{i j} u_{i} \dot{u}_{j}-\frac{\theta}{2} \epsilon_{i j} y_{i} \dot{y}_{j}+\frac{c}{2} u^{2} .
$$

The Euler-Lagrange equations (EOM) which follow from the Lagrangian (42) are

$$
\begin{aligned}
y_{i}-\dot{x}_{i} & =0, & \dot{p}_{i}=0, \quad u_{i}-\dot{y}_{i}=0, \\
p_{i}+\dot{q}_{i}+\theta \epsilon_{i j} \dot{y}_{j} & =0, & q_{i}+\frac{\theta^{\prime}}{4} \epsilon_{i j} \dot{u}_{j}-c u_{i}=0 .
\end{aligned}
$$


Using the Faddeev-Jackiw procedure [9]-[10] we obtain the following nonvanishing Poisson brackets:

$$
\begin{aligned}
& \left\{x_{i}, p_{j}\right\}=\delta_{i j}, \quad\left\{y_{i}, q_{j}\right\}=\delta_{i j}, \\
& \left\{q_{i}, q_{j}\right\}=-\theta \epsilon_{i j}, \quad\left\{u_{i}, u_{j}\right\}=4 \frac{\epsilon_{i j}}{\theta^{\prime}} .
\end{aligned}
$$

To calculate from the Lagrangian (42) the Noether charges we consider the following acceleration extended Galilean transformations for a fixed but arbitrary value of the time parameter $t$,

$$
\begin{array}{ccc}
\delta x_{i}= & -\delta a_{i}-\delta v_{i} t-\delta b_{i} t^{2}+\delta \alpha \epsilon_{i j} x_{j}, \\
\delta y_{i}= & -\delta v_{i}-2 t \delta b_{i}+\delta \alpha \epsilon_{i j} x_{j}, \\
\delta u_{i}= & -2 \delta b_{i}+\delta \alpha \epsilon_{i j} x_{j}, \\
\delta p_{i}= & 2 \theta \epsilon_{i j} \delta b_{j}+\delta \alpha \epsilon_{i j} p_{j}, \\
\delta q_{i}= & -2 c \delta b_{i}+\delta \alpha \epsilon_{i j} q_{j} .
\end{array}
$$

These transformations leave the EOM (43) invariant. The generators which provide the transformations (45) are obtained by the Noether theorem and are given by

$$
\begin{array}{rlrl}
P_{i} & = & p_{i}, \\
K_{i} & = & p_{i} t+q_{i}+\theta \epsilon_{i j} y_{j}, \\
F_{i}= & -p_{i} t^{2}+2 K_{i} t-2 \theta \epsilon_{i j} x_{j}-2 c y_{i}+\frac{\theta^{\prime}}{2} \epsilon_{i j} u_{j}, \\
J & = & \epsilon_{i j} x_{i} p_{j}+\epsilon_{i j} y_{i} q_{j}-\frac{\theta}{2} y^{2}-\frac{\theta^{\prime}}{8} u^{2}
\end{array}
$$

\subsection{Transmutation into a planar model with electro- magnetic interaction}

Note that if we consider the velocities $y_{i}$ as the particle coordinates $X_{i}$ then the model introduced in Section 4.1 would describe the interaction of noncommutative planar exotic particles with dynamical electric fields and a constant magnetic field (see [17], [18]).

Let us perform the substitution:

$$
\begin{gathered}
p_{i} \rightarrow-E_{i}, \quad x_{i} \rightarrow \pi_{i}, \quad y_{i} \rightarrow X_{i}, \quad q_{i} \rightarrow P_{i}, \quad u_{i} \rightarrow Y_{i}, \\
\theta \rightarrow-B, \quad c \rightarrow m, \quad \text { and } \quad \theta^{\prime} \rightarrow-4 \theta m^{2} .
\end{gathered}
$$

Applying the substitution (47) into the Lagrangian (42) we obtain

$$
L=E_{i} X_{i}+\dot{E}_{i} \pi_{i}+P_{i}\left(\dot{X}_{i}-Y_{i}\right)+\frac{\theta m^{2}}{2} \epsilon_{i j} Y_{i} \dot{Y}_{j}+\frac{B}{2} \epsilon_{i j} X_{i} \dot{X}_{j}+\frac{m}{2} Y^{2}
$$


This is the Lagrangian of the higher-order derivative model introduced in [8] supplemented by the standard electromagnetic interaction with dynamical electric field $E_{i}$ and a constant magnetic field $B$ and a kinetic term $\dot{E}_{i} \pi_{i}$ added for the additional phase space variables $E_{i}$ and $\pi_{i}$. In [17], [18] it was shown that such electromagnetic couplings of planar particle models lead to the electromagnetic enlargement of the Galilei symmetry with additional generators given by the electric field $E_{i}$ and with three central charges $(m, \theta$ and $B$ ). It can be checked that the Lagrangian (48) is quasi-invariant under the electromagnetically enlarged $D=(2+1)$ Galilei group with the symmetry algebra which is isomorphic to the acceleration-extended Galilei algebra with three central charges $\left(\theta, \theta^{\prime}, c\right) 6$.

Concluding we see that our massless planar higher order Lagrangian model (40) is clasically equivalent to the Lagrangian for a massive exotic planar point particle interacting with dynamical electric and constant magnetic fields.

\section{$5 \quad$ Final Remarks}

- We would like to add that the extension of the equivalent coordinate frames to the ones with various constant accelerations may have possibly a physical application based on recent developments in astrophysics. If in accordance with recent measurements (see e.g. [18]-[20]) the universe expands with increasing velocity and approximately constant acceleration the coordinate frames extended by constant acceleration motions may be useful in its description

- Note that the Lagrangians invariant under acceleration-extended Galilean symmetries contain higher derivatives. Although such Lagrangians have been discussed in literature (see eg [21], [22]) their physical meaning is still unclear. We would like, however, to point out that following our discussion in Section 4.2 these models might have a meaning in the framework of classical mechanics for exotic particles in $D=(2+1)$ dimensions.

- Analogously to the model introduced by us in [8] (cp. also [23]) one can introduce for our model (40) the phase space decomposition into "external" and "internal" dynamics. This decomposition can be understood as a generalization of the Galilean-invariant decomposition of phase space into the center of mass and relative motion in standard classical mechanics. We plan to treat this method in a general framework

\footnotetext{
${ }^{6}$ In formulae (56-57) we assume that the eletromagnetic coupling constant $e=1$.
} 
and discuss the solutions of the present model and the interpretation of "internal" dynamics for the model with higher time derivatives in a forthcoming publication.

\section{References}

[1] J. Lukierski, P.C. Stichel and W.J. Zakrzewski, Phys. Lett. A 357, 1 (2006); hep-th/0511259.

[2] C.R. Hagen, Phys. Rev. D5, 377 (1972).

[3] U. Niederer, Helv. Phys. Acta 45, 802 (1972).

[4] C. Duval and P.A. Horvathy, J. Math. Phys. 35, 2516 (1994).

[5] V. Bargmann, Ann. Math. 59, 1 (19954).

[6] J.M. Levy-Leblond, in "Group Theory and Applications", (Ed. E. Loebl) Academic Press, New York, 1971.

[7] A. Ballesteros, M, Gadella and A.A. del Olmo, J. Math. Phys. 33, 3379 (1992).

[8] J. Lukierski, P.C. Stichel and W.J. Zakrzewski, Ann. Phys. 260, 224 (1997).

[9] L. Faddeev and R. Jackiw, Phys. Rev. Lett. 60, 1962 (1988)

[10] R. Jackiw in "Constraints Theory and Quantization Methods" (F. Colomo et al., Eds), World Scientific, Singapore, (1994) p. 163

[11] J.M. Souriau, Structure des Systèmes Dynamiques, Ed. Dunod, Paris, 1970

[12] P. Horvathy, Acta Phys. Polon., 34, 2611 (2003).

[13] Y. Brihaye, C. Gonera, S.Giller and P. Kosinski, hep-th/9503046 (unpublished)

[14] C. Duval and P.A. Horvathy, Phys. Lett., B479, 284 (200); J. Phys., A34, 10097 (2001); Phys. Rev. D 43, 3907 (2001)

[15] R. Banerjee, Eur. Phys. J. C 47, 541 (2006) 
[16] P.A. Horvathy, L. Martina and P.C. Stichel, Phys. Lett. B 615, 87 (2005)

[17] M.A. del Olmo, M.S. Plyushchay Ann.Phys. 321, 2830 (2006)

[18] A.G. Riess at al Astron. J. 1161009 (1998); astro-ph/9805201

[19] S. Perlmutter et al Astrophys. J. 517565 (1999); astro-ph/9812133

[20] C.B. Netterfeld et al Astrophys. J. 571604 (2003); astro-ph/0104460

[21] P. Pais and G.E. Uhlenbeck, Phys. Rev. 79, 145 (1950)

[22] Ph.D. Mannheim - hep-th/0608154 (2006)

[23] P.A. Horvathy and M.S. Plyushchay, JHEP 0206, 033 (2002) 